\title{
Avaliação dos hábitos alimentares dos Universitários de uma Instituição Privada de Ensino Superior no interior da Bahia
}

\author{
Marcela Teles Amaral de Souza ${ }^{1}$; Flávio Mendes de Souza. ${ }^{2}$
}

\begin{abstract}
Resumo: A alimentação é uma necessidade básica do ser humano, pois desempenha um papel muito importante durante todo o ciclo de vida dos indivíduos. $\mathrm{O}$ ato de alimentar-se envolve uma multiplicidade de aspectos que influência na qualidade de vida. A alimentação saudável é um dos principais determinantes da saúde que assegura o desenvolvimento e crescimento fisiológico. Os alimentos com alto teor calórico, deterioram o hábito alimentar saudável, o que torna os indivíduos mais suscetíveis a doenças. Este estudo tem como objetivo, avaliar os hábitos alimentares dos universitários de uma instituição privada de ensino superior no interior da Bahia. Trata-se de um estudo do tipo descritivo e transversal, a pesquisa foi realizada com 15 alunos ingressantes, 15 dos semestres medianos e 15 concluintes dos cursos de Estética e Cosmética, Arquitetura e Urbanismo, Enfermagem, Farmácia e Odontologia totalizando 225 alunos do turno vespertino. Os dados foram obtidos através da aplicação de um questionário semiestruturado. Os dados foram tabulados em planilhas do programa Microsoft Office Excel@ 2013, os resultados obtidos de cada variável analisada foram apresentados através de tabelas e gráficos. De maneira geral, poucos alunos trazem lanche de casa para a faculdade, a maioria costuma lanchar nos intervalos das aulas e optam por salgados, a maior parte dos alunos são sedentários e não consideram ter uma alimentação saudável. Diante dos resultados observados, faz-se necessário criar uma estratégia nutricional adequada. Como profissional de saúde, o farmacêutico deve realizar orientações voltadas para alimentação e hábitos de vida saudáveis, com o propósito de controle e prevenção da obesidade, doenças cardiovasculares e outras associadas, visando a formação de atitudes e comportamentos saudáveis que permaneçam por toda vida.
\end{abstract}

Palavras-Chave: Hábitos alimentares. Alimentação saudável. Obesidade. Doenças cardiovasculares.

\section{Evaluation of the eating habits of University students of a Private Higher Education Institution in the interior of Bahia}

\begin{abstract}
Food is a basic human need because it plays a very important role throughout the life cycle of individuals. The act of feeding itself involves a multiplicity of aspects that influence the quality of life. Healthy eating is one of the main determinants of health that ensures the development and physiological growth. Foods with a high caloric content deteriorate the healthy eating habit, which makes the individuals more susceptible to diseases. This study aims to evaluate the eating habits of university students from a private college in the interior of Bahia. It is a descriptive and cross-sectional study, the research was carried out with 15 incoming students, 15 of the medium and 15 concluding semesters Of the Aesthetics and Cosmetics courses, Architecture and Urbanism, Nursing, Pharmacy and Dentistry totaling 45 students of the afternoon shift. The data were obtained through the application of a semi-structured questionnaire. The data were tabulated in spreadsheets of the program Microsoft Office Excel ${ }^{\circledR}$ 2013, the results obtained from each variable analyzed were presented through tables and graphs. Overall, few students bring snacks from home to college, most often snack at school breaks and choose salads, most students are sedentary and do not consider eating healthy. In view of the observed results, it is necessary to create an adequate nutritional strategy. As a health professional, the pharmacist should conduct orientated guidelines for healthy eating and living habits, With the purpose of controlling and preventing obesity, diabetes, cardiovascular and other associated diseases, Aiming at the formation of healthy attitudes and behaviors that remain throughout life.
\end{abstract}

Keywords: Eating habits. Healthy eating. Obesity. Cardiovascular diseases.

\footnotetext{
${ }^{1}$ Faculdade Independente do Nordeste - FAINOR. E-mail: macelamaral@ hotmail.com.

${ }^{2}$ Graduação em Química pela Universidade Estadual de Santa Cruz, UESC, Brasil. Mestrado em Quimica pela Universidade Estadual do Sudoeste da Bahia, UESB, Brasil.
} 
Id on Line Revista Multidisciplinar e de Psicologia

Id on Line Multidisciplinary and Psycology Journal

\section{Introdução}

A alimentação é uma necessidade básica do ser humano, pois desempenha um papel muito importante durante todo o ciclo de vida dos indivíduos. $\mathrm{O}$ ato de alimentar-se envolve uma multiplicidade de aspectos que influência na qualidade de vida. Uma alimentação saudável, associada à prática de atividades físicas constituem requisitos fundamentais para prevenção de algumas doenças debilitantes. (MUNIZ, 2013; QUEIROZ et al, 2015).

A alimentação é muito importante durante a infância e a adolescência, para o crescimento e desenvolvimento e representa um dos principais fatores de prevenção para algumas doenças na idade adulta. Há evidências de que algumas patologias típicas do adulto, como doenças cardíacas, obesidade, osteoporose e câncer ocorrem devido à exposição aos fatores de risco, entre eles, a dieta, em diferentes fases da vida. (GIBNEY, 2005).

Devido ao ritmo de vida atual, com pouco tempo para dedicar-se as refeições saudáveis, a população jovem adota hábitos alimentares desequilibrados, pois os mesmos substituem os alimentos saudáveis pelo consumo de alimentos hipercalóricos, com excesso de gorduras e açúcares. Essas mudanças na alimentação associadas a um estilo de vida sedentário levam ao acúmulo de excesso de gordura, ao surgimento do sobrepeso e consequentemente a obesidade e uma série de doenças debilitantes. (DANELON et al, 2006).

A alimentação saudável é um dos principais determinantes da saúde que assegura o desenvolvimento e crescimento fisiológico. Os alimentos com alto teor calórico, deterioram o hábito alimentar saudável, o que torna os indivíduos mais suscetíveis a doenças. (GIBNEY, 2005).

Dentre as doenças causadas por distúrbios alimentares a obesidade é a que mais tem se destacado, pelo fato de atingir um elevado número de pessoas. A obesidade é uma Doença Crônica Não Transmissível (DCNT), considerada um distúrbio nutricional. De maneira simplificada, a obesidade é definida como uma doença em que o acúmulo de gordura corporal em excesso afeta a saúde dos indivíduos. Seu surgimento está relacionado com maus hábitos alimentares da população. (MEDINA et al, 2005).

O investimento em medidas de saúde no âmbito escolar é uma ferramenta segura e eficiente para combater distúrbios nutricionais, essas medidas incluem estímulos aos alunos a 
Id on Line Revista Multidisciplinar e de Psicologia

Id on Line Multidisciplinary and Psycology Journal

prática de atividade física, mudanças nos hábitos alimentares e informações sobre questões nutricionais. (VIUNISKI, 2005).

A faculdade é um local onde programas de educação e saúde, podem ter grande repercussão e influenciar os jovens a mudar os hábitos alimentares atingindo os universitários nas etapas influenciáveis de suas vidas, pois é na faculdade que muitos alunos fazem suas refeições, realizando suas preferências e hábitos alimentares. (DAMELON, et al 2006).

O consumo excessivo de alimentos com grande densidade energética é muito preocupante, pois podem levar os indivíduos a desenvolverem uma série de doenças. Portanto uma alimentação saudável é fundamental para garantir uma boa saúde e qualidade de vida. (FELICE et al, 2007).

Diante do que foi descrito e considerando a importância da realização de estudos sobre o consumo alimentar da população jovem, o presente estudo tem por objetivo avaliar os hábitos alimentares dos alunos de uma instituição privada de ensino superior no interior do estado da Bahia.

\section{Metodologia}

A pesquisa foi realizada na Faculdade Independente do Nordeste (FAINOR), localizada no município de Vitória da Conquista, situado no interior Bahia. O estudo realizado foi do tipo descritivo e transversal, com abordagem qualitativa. A pesquisa foi realizada com 15 alunos ingressantes, 15 dos semestres medianos e 15 concluintes dos cursos de Estética e Cosmética, Arquitetura e Urbanismo, Enfermagem, Farmácia e Odontologia, totalizando 45 alunos do turno vespertino.

O consumo alimentar foi avaliado de forma qualitativa, para tal avaliação foi utilizado um questionário semiestruturado com perguntas relacionadas a qualidade alimentar. Os questionários foram aplicados em dois dias no período da tarde, os participantes da pesquisa receberam o TCLE, um termo de consentimento que continha todas as informações da pesquisa. O questionário e o termo de consentimento estão em anexo, (anexo I e II). Os dados obtidos através dos questionários foram tabulados em planilhas do programa Microsoft Office Excel® 
2013, e os resultados obtidos de cada variável analisada foram apresentados através de tabelas e gráficos.

\section{Resultados e Discussão}

Os questionários foram respondidos por 225 universitários, sendo 15 alunos ingressantes, 15 dos semestres medianos e 15 concluintes dos cursos de Estética e Cosmética, Arquitetura e Urbanismo, Enfermagem, Farmácia e Odontologia. As respostas dos questionários foram descritas e comparadas em cada curso. A pesquisa foi realizada com os alunos de ambos os sexos, sendo $185(82,2 \%)$ do sexo feminino e $40(17,8 \%)$ do sexo masculino. As alunas demonstraram maior interesse tanto em participar da pesquisa, como em responder as perguntas do questionário.

Observou-se que no período inicial de cada curso os entrevistados possuem entre 17 a 25 anos. Os universitários dos semestres medianos, bem como os concluintes possuem entre 21 a 25 anos e algumas pessoas entre 26 ou mais de 30 anos de idade.

Segundo o gráfico 1, o curso de estética e cosmética é o que possui a maior quantidade de alunos bolsistas (33\%), seguido de farmácia e enfermagem (22\%), odontologia $(17,8 \%)$ e arquitetura e urbanismo (9\%), que apresenta o menor índice de estudantes com bolsas de estudo. A diferença nos percentuais de bolsas entre os cursos pode ser explicada pela renda familiar, visto que, os universitários com o menor número de bolsas são os que possuem maior renda em detrimento aos alunos dos demais cursos, o que pode ser observado no gráfico 2 . 
Id on Line Revista Multidisciplinar e de Psicologia

Id on Line Multidisciplinary and Psycology Journal

Gráfico 1: Referente a bolsa de estudos

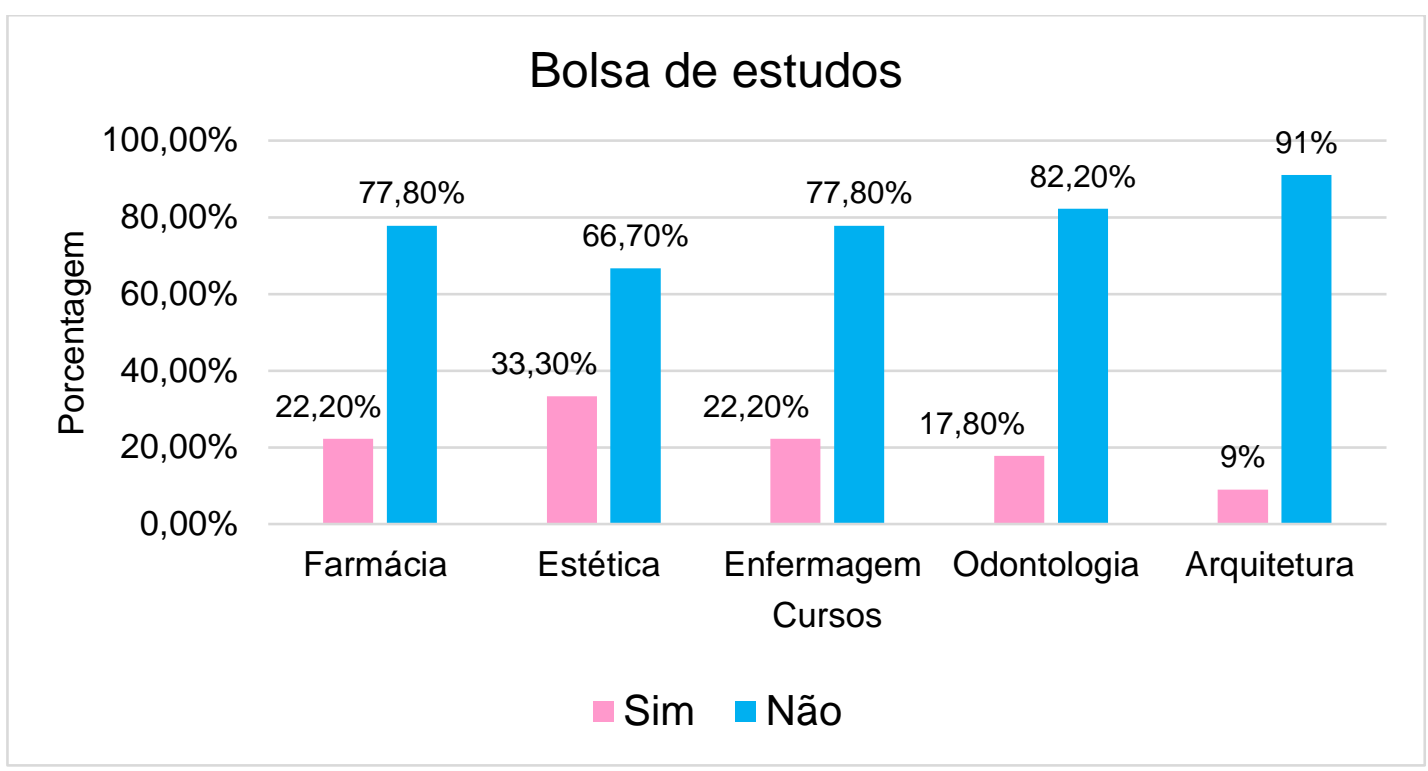

Fonte: Dados da pesquisa, 2016.

Gráfico 2: Renda familiar

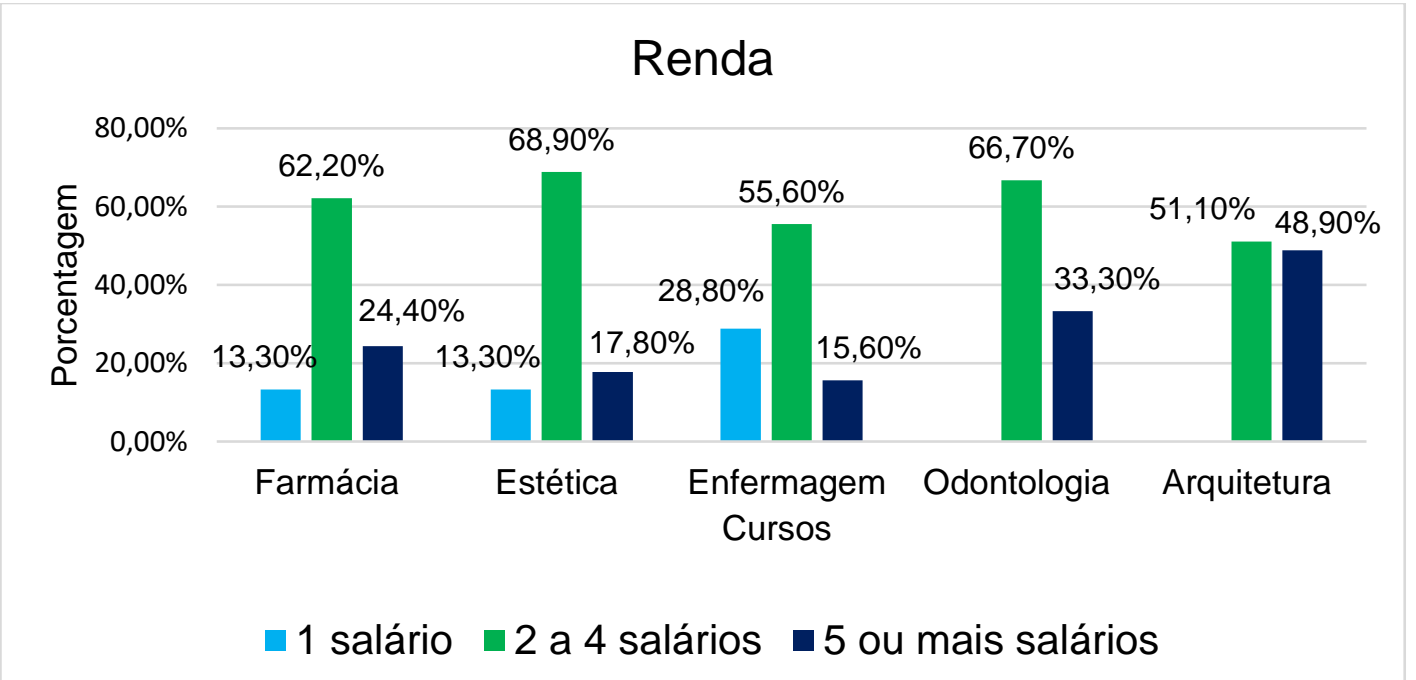

Fonte: Dados da pesquisa, 2016.

No gráfico 3, verifica-se a frequência das refeições realizadas durante o dia incluindo os lanches, nota-se que a maioria dos alunos se alimenta de uma a quatro vezes por dia. 
Id on Line Revista Multidisciplinar e de Psicologia

Id on Line Multidisciplinary and Psycology Journal

Gráfico 3: Frequência de refeições realizadas durante o dia incluindo os lanches

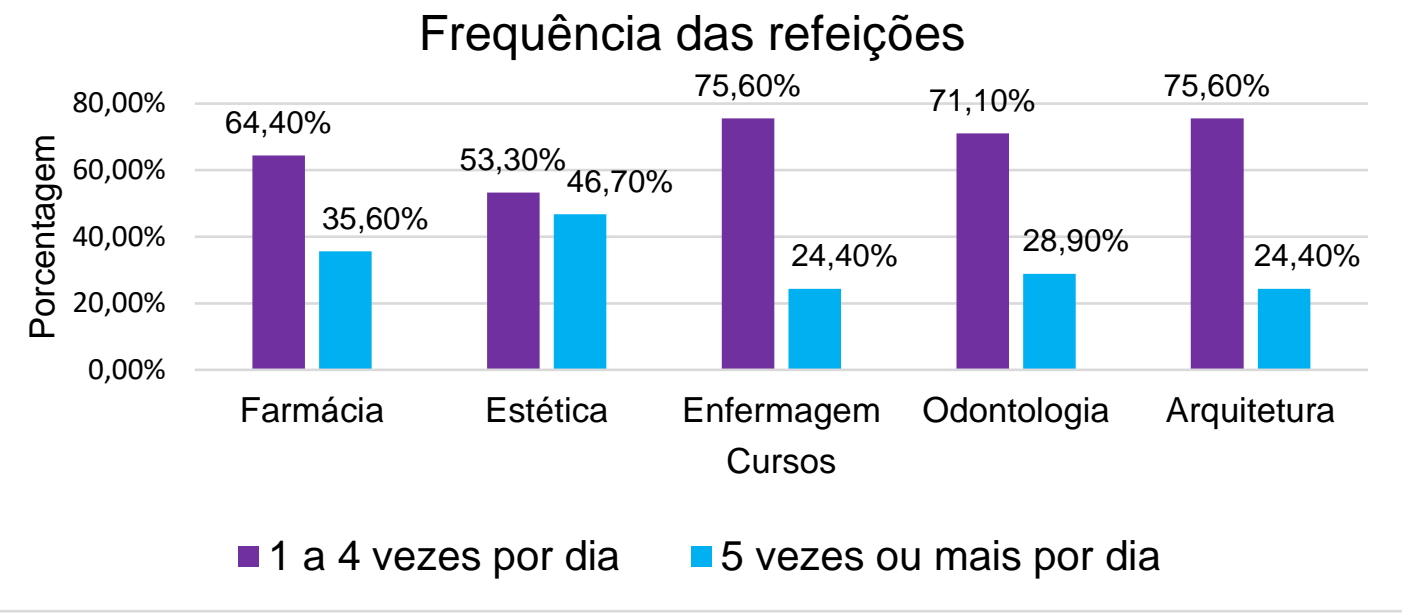

Fonte: Dados da pesquisa, 2016.

A tabela 1 apresenta o consumo de lanches entre os alunos, podendo ser observado que não ocorreram diferenças significantes entre os cursos avaliados, pois a maioria dos estudantes consomem lanches nos intervalos das aulas.

Tabela 1: Consumo de lanches nos intervalos das aulas.

$\begin{array}{llllll}\text { Frequência } & \text { Farmácia } & \text { Estética } & \text { Enfermagem } & \text { Odontologia } & \text { Arquitetura } \\ \text { Sim } & 66,7 \% & 71,1 \% & 77,8 \% & 66,7 \% & 77,8 \% \\ \text { Não } & 33,3 \% & 28,9 \% & 22,2 \% & 33,3 \% & 22,2 \% \\ \text { TOTAL } & \mathbf{1 0 0 \%} & \mathbf{1 0 0 \%} & \mathbf{1 0 0 \%} & \mathbf{1 0 0 \%} & \mathbf{1 0 0 \%}\end{array}$

Fonte: Dados da pesquisa, 2016.

De acordo a tabela 2, no curso de estética e cosmética os alunos consomem maior quantidade de frutas (24\%), os demais optam por salgados no horário do lanche, sendo o maior consumo realizado pelos universitários de enfermagem, seguido pelos de arquitetura e urbanismo (80\%), odontologia (73\%) e farmácia $(71 \%)$. 
Id on Line Revista Multidisciplinar e de Psicologia

Id on Line Multidisciplinary and Psycology Journal

Tabela 2: Tipos de alimentos consumidos na hora do lanche.

\begin{tabular}{r|lllll} 
Alimentos & Farmácia & Estética & Enfermagem & Odontologia & Arquitetura \\
Frutas & $8,9 \%$ & $24,4 \%$ & $4,4 \%$ & $8,9 \%$ & $8,9 \%$ \\
Salgados & $71,1 \%$ & $62,2 \%$ & $82,2 \%$ & $73,3 \%$ & $80 \%$ \\
Outros & $20 \%$ & $13,3 \%$ & $13,3 \%$ & $17,8 \%$ & $11,1 \%$ \\
TOTAL & $\mathbf{1 0 0 \%}$ & $\mathbf{1 0 0 \%}$ & $\mathbf{1 0 0 \%}$ & $\mathbf{1 0 0 \%}$ & $\mathbf{1 0 0 \%}$
\end{tabular}

Fonte: Dados da pesquisa, 2016.

Nos cursos de estética e cosmética e arquitetura há uma maior prevalência pelo consumo de salgados assados, apresentando um percentual de $60 \%$ e $51 \%$ respectivamente. Enquanto nos demais cursos a preferência é maior por salgados fritos, o que pode ser observado no gráfico 4.

Gráfico 4: Tipos de salgados preferidos pelos universitários.

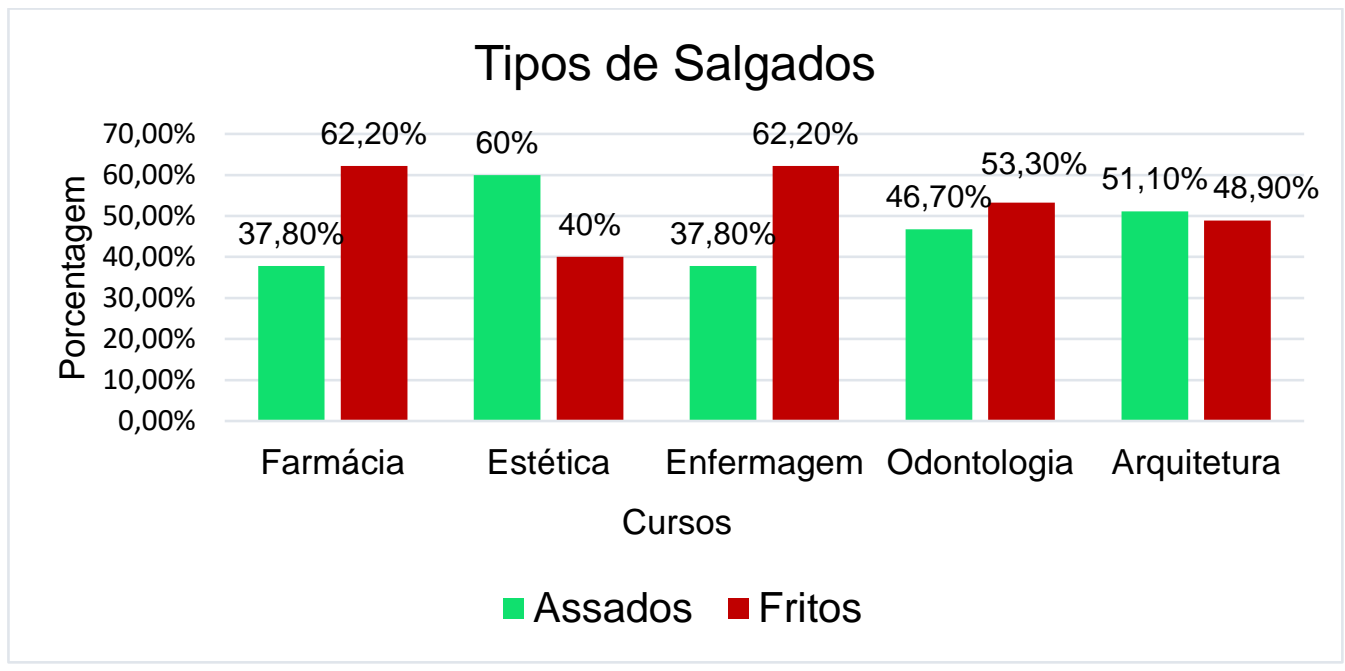

Fonte: Dados da pesquisa, 2016.

O gráfico 5, mostra que entre os alimentos mais consumidos a coxinha e o pastel de forno tiveram maior destaque em todos os cursos, alimentos estes com alto teor de gorduras, do ponto de vista nutricional esses alimentos apresentam vários riscos potenciais a saúde, pois o consumo excessivo de ácidos graxos, tem sido associado ao desenvolvimento de vários fatores 
Id on Line Revista Multidisciplinar e de Psicologia

Id on Line Multidisciplinary and Psycology Journal

de risco que podem causar doenças crônicas e inúmeros prejuízos a saúde das pessoas, causando grande impacto aos níveis de colesterol, deprimindo o sistema imunológico e contribuindo para a formação de placas de ateromas o que favorecem o desenvolvimento de doenças cardiovasculares, e outras associadas (LAMOUNIER, 2008).

Gráfico 5: Salgados consumidos com maior frequência pelos estudantes

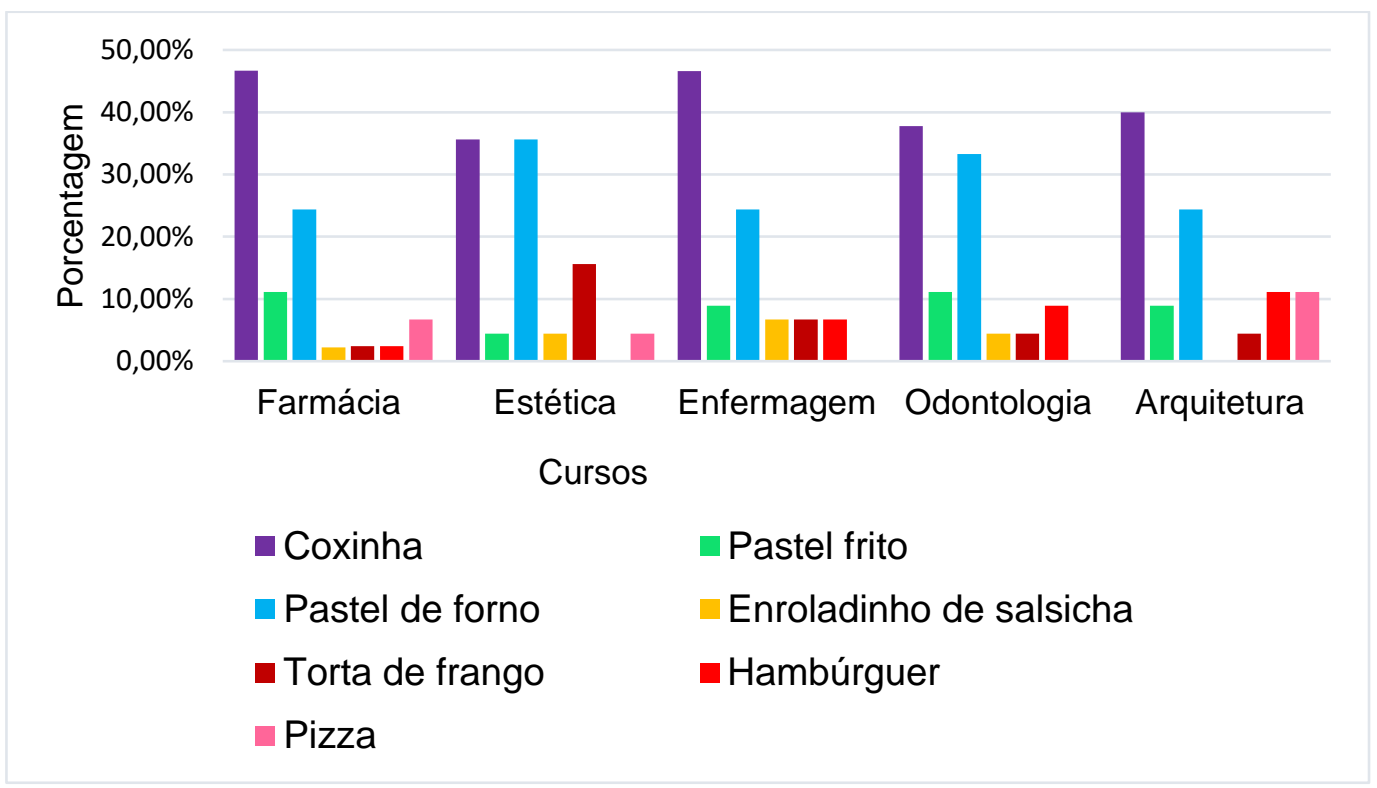

Fonte: Dados da pesquisa, 2016.

Na tabela 3, nota-se que todos os alunos entrevistados relataram ter acesso a informações referentes à alimentação e hábitos de vida saudáveis, entretanto grande parte dos mesmos afirmam não possuir uma alimentação saudável, o que pode ser observado na tabela 4, evidenciando-se assim que o acesso a informações não garante uma qualidade de vida adequada.

Tabela 3: Quantidade de alunos com acesso a informações sobre alimentação e hábitos de vida saudáveis

$\begin{array}{llllll}\text { Frequência } & \text { Farmácia } & \text { Estética } & \text { Enfermagem } & \text { Odontologia } & \text { Arquitetura } \\ \text { Sim } & 45 & 45 & 45 & 45 & 45 \\ \text { Não } & 0 & 0 & 0 & 0 & 0 \\ \text { TOTAL } & \mathbf{4 5} & \mathbf{4 5} & \mathbf{4 5} & \mathbf{4 5} & \mathbf{4 5}\end{array}$

Fonte: Dados da pesquisa, 2016. 
Id on Line Revista Multidisciplinar e de Psicologia

Id on Line Multidisciplinary and Psycology Journal

Tabela 4: Quantidade de alunos que consideram ter uma alimentação saudável

\begin{tabular}{|llllll|}
\hline Frequência & Farmácia & Estética & Enfermagem & Odontologia & Arquitetura \\
Sim & 18 & 14 & 5 & 12 & 9 \\
Não & 27 & 31 & 40 & 33 & 36 \\
TOTAL & $\mathbf{4 5}$ & $\mathbf{4 5}$ & $\mathbf{4 5}$ & $\mathbf{4 5}$ & $\mathbf{4 5}$ \\
& & & & & \\
\hline
\end{tabular}

Fonte: Dados da pesquisa, 2016.

De acordo a tabela 5, a maioria dos universitários são sedentários, não praticam nenhum tipo de atividade física, o que torna os alunos mais susceptíveis a doenças associadas a maus hábitos de vida.

Tabela 5: Frequência da prática de atividade física por semana

\begin{tabular}{l|lllll}
\multicolumn{1}{c|}{ Frequência } & Farmácia & Estética & Enfermagem & Odontologia & Arquitetura \\
\hline $\begin{array}{l}\text { Apenas 1 dia } \\
\text { 2 a 4 vezes } \\
\text { por dia }\end{array}$ & 6 & 2 & 3 & 4 & 2 \\
$\begin{array}{l}\text { Todos os } \\
\text { dias }\end{array}$ & 9 & 12 & 11 & 8 & 9 \\
Não pratica & 26 & 5 & 4 & 6 & 5 \\
$\quad$ TOTAL & $\mathbf{4 5}$ & $\mathbf{4 5}$ & $\mathbf{4 5}$ & 27 & 29 \\
\end{tabular}

Fonte: Dados da pesquisa, 2016.

Segundo a tabela 6, todos os alunos entrevistados consideram importante mudar os hábitos alimentares, entretanto a maioria possui uma alimentação inadequada e hábitos de vida prejudiciais à saúde. 
Id on Line Revista Multidisciplinar e de Psicologia

Id on Line Multidisciplinary and Psycology Journal

Tabela 6: Frequência dos alunos que consideram importante modificar os hábitos alimentares.

$\begin{array}{llllll}\text { Frequência } & \text { Farmácia } & \text { Estética } & \text { Enfermagem } & \text { Odontologia } & \text { Arquitetura } \\ \text { Sim } & 45 & 45 & 45 & 45 & 45 \\ \text { Não } & 0 & 0 & 0 & 0 & 0 \\ \text { TOTAL } & \mathbf{4 5} & \mathbf{4 5} & \mathbf{4 5} & \mathbf{4 5} & \mathbf{4 5}\end{array}$

Fonte: Dados da pesquisa, 2016.

Os alimentos vendidos pelas cantinas da faculdade são de alto valor calórico e baixo valor nutritivo com alta concentração lipídica, o que contribui para o desenvolvimento da obesidade e outras doenças associadas. Sabe-se que teores altos de lipídios podem provocar consequência metabólica no organismo, sendo prejudicial à saúde (BISMARCKNASR et al., 2006).

Consequentemente, o consumo excessivo de alimentos ricos em gorduras pode causar sérios riscos à saúde, como a elevação do nível de colesterol total e do LDL (Colesterol ruim), redução dos níveis de HDL (Colesterol bom), aumento dos níveis de triacilgliceróis no plasma sanguíneo. Dessa forma, verifica-se os efeitos deletérios que o consumo da gordura trans pode causar à saúde humana, permitindo classificá-la como a mais nociva de todas as gorduras (LAMOUNIER, 2008).

Como já foi destacado na descrição dos resultados, os cursos de Odontologia e Arquitetura e Urbanismo são os que os alunos possuem maior renda familiar, porém não possuem alimentação e hábitos de vida saudáveis. Neste contexto nota-se que a renda não influência na qualidade da alimentação. Apenas no curso de Estética e cosmética observa-se que entre os entrevistados $24 \%$ preferem frutas na hora do lanche, o que demonstra que possuem uma melhor qualidade na alimentação em relação aos demais cursos.

De maneira geral, poucos estudantes trazem lanche de casa para a faculdade, a maioria costuma lanchar nos intervalos das aulas e optam por salgados, além disso, grande parte dos alunos são sedentários. Nota-se que a maioria dos discentes tem acesso a informações sobre alimentação e hábitos de vida saudáveis e consideram importante mudar os hábitos alimentares, porém é perceptível que os mesmos não utilizam as informações adquiridas de forma adequada. Esses resultados são uma tendência observada no Brasil e em várias regiões do mundo, como 
Id on Line Revista Multidisciplinar e de Psicologia

Id on Line Multidisciplinary and Psycology Journal

reflexo de demandas geradas pelo novo modo de vida urbana, caracterizado por escassez de tempo, vasta oferta de produtos, baixa flexibilização dos horários das refeições e grande apelo publicitário em torno dos alimentos (COSTA et al, 2005).

\section{Conclusão}

Este trabalho permitiu identificar e avaliar o consumo alimentar dos universitários ingressantes, medianos e concluintes dos cursos de Arquitetura e Urbanismo, Estética e Cosmética, Enfermagem, Farmácia e Odontologia na Faculdade Independente do Nordeste. Os resultados encontrados nesse estudo mostram que os alimentos mais consumidos são os que possuem maior concentração de gordura e açúcar e são extremamente prejudiciais à saúde.

Verificou-se que os universitários participantes da pesquisa apresentam um padrão de consumo alimentar muito semelhante no que se refere aos tipos de alimentos comprados nas cantinas da faculdade e as preferencias alimentares.

Diante dos resultados observados, faz-se necessário criar uma estratégia nutricional adequada. Como profissional de saúde, o farmacêutico deve realizar orientações voltadas para alimentação e hábitos de vida saudáveis, com o propósito de controle e prevenção da obesidade, diabetes, doenças cardiovasculares e outras associadas, visando a formação de atitudes e comportamentos saudáveis que permaneçam por toda vida.

\section{Referências}

COSTA, T. H. M; SCHIMITZ, B. A. S.; TUMA, R. C. F. B. Avaliação antropométrica e dietética de pré-escolares em três creches de Brasília, Distrito Federal. Revista Brasileira Saúde Materno Infantil, v. 5, n. 4, out./dez. 2005.

BISMARCK-NASR, E. M.; FRUTUOSO, M. F. P.; GAMBARDELLA, A. M. D. Importância nutricional dos lanches na dieta de adolescentes urbanos de classe média. Pediatria (São Paulo), v.28, n.1, p.26-32, 2006.

DANELON, Maria Angélica Schievano. Serviços de alimentação destinados ao público escolar: análise da convivência do Programa de Alimentação Escolar e das cantinas. Segurança Alimentar e Nutricional, Campinas, 13(1): 85-94, 2006. 
Id on Line Revista Multidisciplinar e de Psicologia

Id on Line Multidisciplinary and Psycology Journal

FELICE, MARINA MARQUART, et al. Análise do Lanche Escolar Consumido por Adolescentes. SAÚDE REV., Piracicaba, 9 (22): 7-14, 2007.

GIBNEY, M.J. et al. Introdução a nutrição humana. Rio de Janeiro: Guanabara Koogan, 2005 .

LAMOUNIER, R. A gordura trans, seus riscos e a proibição em Nova Iorque: paranóia ou prevenção? Disponível em: http://www.diabetes.org.br. Acesso em 16 de Outubro de 2016.

MEDINA, José Luís et al. Programa Nacional de combate à obesidade. Acesso em: 17 de outubro de 2016. Disponível em: http://1nj5ms2lli5hdggbe3mm7ms5.wpengine.netdnacdn.com/files/2015/08/Programa-Nacional-de-Combate-\%C3\%A0-Obesidade.pdf.

MESQUITA, Jussara Helou, et al. Perfil qualitativo dos lanches escolares consumidos em instituição de ensino particular do Distrito Federal - Brasil. Universitas: Ciências da Saúde, v. 4, n. 1 / 2, p. 49-62, 2006.

MUNIZ, Ludmila Correa et al. Prevalência e fatores associados ao consumo de frutas, legumes e verduras entre adolescentes de escolas públicas de Caruaru,PE. Ciênc. saúde coletiva [online]. 2013, vol.18, n.2, pp. 393-404.

QUEIROZ, Aline Bianca Lisboa, et al. Consumo alimentar e percepção de imagem corporal entre adolescentes de escola privada. Unijuí. Rio Grande do Sul. 20015.

VIUNISKI N. Obesidade em adultos, um desafio pediátrico. Rev. Nutrição em pauta, n. 74, p. 4-10, set/out. 2005.

Como citar este artigo (Formato ABNT):

SOUZA, M.T.A.; SOUZA, F.M. Avaliação dos hábitos alimentares dos Universitários de uma Instituição Privada de Ensino Superior no interior da Bahia. Id on Line Revista Multidisciplinar e de Psicologia, Janeiro de 2017, vol.10, n.33, p. 262-273. ISSN: 1981-1179.

Recebido: 02/12/2016

Aceito: $02 / 12 / 2016$ 